\title{
A. Erstes Kapitel: Übergreifende Verfassungsfragen und Probleme
}

\section{Gesetzgebungskompetenzen}

Der in den Kompetenzvorschriften des Grundgesetzes verwendete Begriff des Strafvollzuges schließt den Maßregelvollzug ein.

DSo wie die $\S \S 63,64$ StGB dem "Strafrecht" im Sinne des Art. 74 Nr. 1 GG zuzuordnen sind, so gehört ihr Vollzug als Vollzug der Freiheitsentziehung auf Grund strafgerichtlicher Urteile zum Strafvollzug in dem Sinne, in dem dieser Begriff in Art. 74 Nr. 1 GG verwendet wird. Der Begriff "Strafvollzug" wird daher herkömmlich als ein Synonym für "Straf- und Maßregelvollzug" verstanden (...). Demgemäß hat der Bund zur Regelung des Vollzugs der freiheitsentziehenden Maßregeln der Besserung und Sicherung die Kompetenz zur konkurrierenden Gesetzgebung (...). Den Ländern steht die Gesetzgebungsbefugnis für diesen Gegenstand gemäß Art. 72 Abs. 1 i.V.m. Art. 74 Nr. 1 GG nur $\mathrm{zu}$, solange und soweit der Bund von seinem Gesetzgebungsrecht keinen Gebrauch macht."1

Die Gesetzgebungskompetenz für den Strafvollzug bezieht sich danach grundsätzlich auch auf den Vollzug der Sicherungsverwahrung. Ungeachtet des Übergangs der Gesetzgebungskompetenz für den Strafvollzug auf die Länder im Jahr 2006 (s. Art. 72 Abs. 1 i.V.m. Art. 74 Abs. 1 Nr. 1 GG) wird jedoch der Bundesgesetzgeber als zuständig angesehen, auf der Grundlage seiner Gesetzgebungskompetenz für das Strafrecht (Art. 74 Abs. 1 Nr. 1 GG) die wesentlichen Leitlinien für die Ausgestaltung der Sicherungsverwahrung vorzugeben.

- „Aus Sicht des Freiheitsschutzes spielt es insoweit keine Rolle, dass der Bundesgesetzgeber seit der Föderalismusreform im Jahr 2006 nicht mehr über die Gesetzgebungskompetenz für den Strafvollzug verfügt.

1 BVerfGE 85, 134 (142); dort auch zur Kompetenzkonformität der damaligen Regelung des $\S 10$ der Justizverwaltungskostenordnung und zur Kompetenzwidrigkeit davon abweichender landesrechtlicher Regelungen. 
Wenn er sich im Rahmen seiner Gesetzgebungskompetenz für das Strafrecht aus Art. 74 Abs. 1 Nr. 1 GG für ein zweispuriges Sanktionensystem und den Einsatz einer so einschneidenden freiheitsentziehenden Maßnahme wie der Sicherungsverwahrung entscheidet, muss er die wesentlichen Leitlinien des freiheitsorientierten und therapiegerichteten Gesamtkonzepts, das der Sicherungsverwahrung von Verfassungs wegen zugrundezulegen ist, selbst regeln und sicherstellen, dass diese konzeptionelle Ausrichtung der Sicherungsverwahrung nicht durch landesrechtliche Regelungen unterlaufen werden kann. //

Bundes- und Landesgesetzgeber stehen gemeinsam in der Pflicht, ein normatives Regelungskonzept zu schaffen, welches den dargelegten Anforderungen genügt. Ihre Aufgabe ist es, unter Berücksichtigung des verfassungsrechtlichen Kompetenzgefüges ein freiheitsorientiertes und therapiegerichtetes Gesamtkonzept der Sicherungsverwahrung zu entwickeln. Dabei ist der Bundesgesetzgeber angesichts seiner konkurrierenden Gesetzgebungszuständigkeit für den Bereich des Strafrechts nach $\S 74$ Abs. 1 Nr. 1 GG darauf beschränkt - aber, wenn er am Institut der Sicherungsverwahrung grundsätzlich festhalten will, auch gehalten - die wesentlichen Leitlinien vorzugeben. Vorgaben in diesem Sinn finden sich etwa in $\S 2$ ThUG. Darüber hinaus ist er zuständig für die Regelungen zur gerichtlichen Überprüfung der Fortdauer der Sicherungsverwahrung und für Verfahrensvorschriften. Die Landesgesetzgeber wiederum haben im Rahmen ihrer Gesetzgebungszuständigkeit das Abstandsgebot sichernde, effektive Regelungen für den Vollzug der Maßregel zu treffen, die einen freiheitsorientierten und therapiegerichteten Vollzug gewährleisten. Dabei ist vor allem sicherzustellen, dass die genannten Anforderungen nicht durch Gewährung zu weiter Spielräume in der Praxis umgangen werden können und damit das Abstandsgebot faktisch leerläuft. Ohne Wahrung des Abstandsgebots ist das Institut der Sicherungsverwahrung mit dem Freiheitsgrundrecht der Untergebrachten nicht vereinbar.“2

Eine Vorlage im Verfahren der konkreten Normenkontrolle (Art. 100 Abs. 1 GG), die die Reichweite der auf die Länder übergegangenen Regelungskompetenz für den Untersuchungshaftvollzug betraf, hat das Bundesverfassungsgericht für mangels ausreichender Darlegung der Entscheidungserheblichkeit unzulässig erachtet (BVerfG II/3, Beschluss vom

2 BVerfGE 128, 326 (387f.). 
20.11.2008 - 2 BvL 16/08 -, juris, betr. Regelung der Gerichtszuständigkeit für die Textkontrolle des Schriftverkehrs). Über die Frage, ob eine staatsvertragliche und landesgesetzliche Maßgabe mit dem Grundgesetzt vereinbar ist, wonach für eine vom Land Berlin auf brandenburgischem Gebiet betriebene Justizvollzugsanstalt das Vollzugsrecht des Landes Berlin anzuwenden ist, konnte wegen Unzulässigkeit der diese Frage aufwerfenden Verfassungsbeschwerde ebenfalls nicht entschieden werden (BVerfG II/3, Beschluss vom 14.8.2013 - 2 BvR 1601/13 -, juris).

II. Erfordernis gesetzlicher Regelung („Gesetzesvorbehalt“)

Der Vorbehalt des Gesetzes, d.h. das - unabhängig vom Vorliegen guter inhaltlicher Eingriffsgründe bestehende - Erfordernis einer gesetzlichen Grundlage für Grundrechtseingriffe, gilt auch für den Strafvollzug, einschließlich des Jugendstrafvollzuges,

- „Eingriffe in Grundrechte bedürfen einer gesetzlichen Grundlage. Seit 1972 ist geklärt, dass von diesem Erfordernis auch Eingriffe in die Grundrechte von Strafgefangenen nicht ausgenommen sind (BVerfGE 33, 1 [9f.]; vgl. auch BVerfGE 58, 358 [367]). Grundrechtseingriffe, die über den Freiheitsentzug als solchen hinausgehen, bedürfen danach unabhängig von den guten oder sogar zwingenden sachlichen Gründen, die für sie sprechen mögen, einer eigenen gesetzlichen Grundlage, die die Eingriffsvoraussetzungen in hinreichend bestimmter Weise normiert (vgl. BVerfGE 40, 276 [283]). //Es gibt keinen Grund, weshalb für den Jugendstrafvollzug etwas anderes gelten sollte. “3

„Die Grundrechte des Strafgefangenen unterliegen zwar erheblich weiter gehenden Einschränkungen als die Grundrechte von Personen in Freiheit, weil und soweit es für solche Einschränkungen rechtfertigende sachliche Gründe gibt. Das ändert aber nichts an der Grundrechtsträgerschaft des Gefangenen und den sich daraus ergebenden Anforde-

3 BVerfGE 116, 69 (80 f.); für Verfahren, mit denen das Fehlen einer gesetzlichen Grundlage für den Jugendstrafvollzug zunächst, überwiegend in vollstreckungsrechtlichem Zusammenhang, erfolglos zur Prüfung zu stellen versucht worden war, s. BVerfG II/2, Beschluss vom 4.5.1994 - 2 BvL 22/91 -, juris; BVerfG II/2, Beschluss vom 8.12.1994 - 2 BvR 2250/94 -, juris; BVerfG II/1, Beschluss vom 9.5.2006 - 2 BvL 1/02 -, juris. 
rungen an den gerichtlichen Rechtsschutz gegen Grundrechtseingriffe. In seiner Eigenschaft als Träger von Grundrechten unterscheidet der Strafgefangene sich von anderen weder dadurch, dass seine Grundrechte von vornherein einen engeren Schutzbereich hätten, noch hinsichtlich des Erfordernisses einer gesetzlichen Grundlage und ausreichender rechtfertigender Gründe für Eingriffe in // seine Grundrechte (vgl. BVerfGE 33, $1<11 \mathrm{ff} .>$ ). Das Erfordernis einer gesetzlichen Grundlage für solche Eingriffe besteht unabhängig von den guten oder sogar zwingenden sachlichen Gründen, die für den Eingriff sprechen mögen (Urteil des Zweiten Senats des Bundesverfassungsgerichts vom 31. Mai 2006 - 2 BvR 1673/04 und 2 BvR 2402/04 -, NJW 2006, S. $2093<2094>$ ). “4

\section{sowie für den Maßregelvollzug}

- „Jede Maßnahme, die mit einem Grundrechtseingriff verbunden ist, bedarf einer Ermächtigungsgrundlage, aus der sich in einer dem rechtsstaatlichen Gebot der Normenklarheit entsprechenden Weise die Eingriffsvoraussetzungen und der Umfang der erlaubten Eingriffe ergeben (vgl. BVerfGE 65, $1<44>$; 113, $29<50>$; BVerfG, Beschluss der 2. Kammer des Zweiten Senats vom 30. November 2006 - 2 BvR 1418/05 -, NStZ-RR 2007, S. $92<93>$ ). ... Diese Anforderung gilt allgemein und unabhängig von den guten oder sogar zwingenden sachlichen Gründen, die für den Eingriff sprechen mögen; eingreifende Maßnahmen im Straf- und im Maßregelvollzug sind von ihr nicht ausgenommen (vgl. BVerfGE 116, $69<80>$; BVerfG, Beschlüsse der 2. Kammer des Zweiten Senats vom 30. August 2006 - 2 BvR 1803/05 -, juris, und vom 30 . November 2006, a.a.O.; ...). ${ }^{\text {"5 }}$

4 BVerfGK 9, 123 (126 f.); zum Erfordernis einer gesetzlichen Grundlage für Eingriffe im Strafvollzug auch BVerfGE 89, 315 (322f.); BVerfGK 19, 140 (146f.); BVerfGK 2, 102 (105); BVerfG II/3, Beschluss vom 10.7.2013 - 2 BvR 2815/11 -, juris.

5 BVerfGK 12, 402 (407); betr. Ahndung von Regelverstößen im Maßregelvollzug; s. auch BVerfG II/2, Beschluss vom 12.3.2008 - 2 BvR 2219/06 -, juris, betr. Verknüpfung einer Besuchserlaubnis im Maßregelvollzug mit der Bedingung eines „Erstgesprächs“ zwischen Besucher und Therapeut, Auszug weiter unten. 
und den Untersuchungshaftvollzug.

- „In Grundrechte darf nur auf gesetzlicher Grundlage eingegriffen werden. Dieser allgemeine rechtsstaatliche Grundsatz gilt auch für den Vollzug der Untersuchungshaft. "6

Eine Rechtfertigung von Eingriffen, für die eine gesetzliche Grundlage fehlt, mit der Rechtsfigur des „besonderen Gewaltverhältnisses“ scheidet aus.

- „Auch die Grundrechte von Strafgefangenen können nur durch Gesetz oder aufgrund eines Gesetzes eingeschränkt werden (BVerfGE 33, 1 [10f.]; st. Rspr.). Es ist daher ausgeschlossen, bei der Strafaussetzung solche Einschränkungen aus einem besonderen Gewaltverhältnis zu begründen."7

Soweit es danach für den Strafvollzug allgemein sowie für den Jugendstrafvollzug zunächst an gesetzlichen Grundlagen fehlte, waren eingreifende Maßnahmen jeweils auch nach den entsprechenden Feststellungen des Bundesverfassungsgerichts noch jeweils für eine Übergangsfrist mit näheren Maßgaben hinzunehmen (s. vor Inkrafttreten des Stravollzugsgesetzes BVerfGE 33, $1<13>$; 40, $276<283>$; BVerfGE 41, $329<330$ f.>; vor Inkrafttreten gesetzlicher Regelungen zur Ausgestaltung des Jugendstrafvollzuges BVerfGE 116, $69<93>$ ).

Das Erfordernis einer gesetzlichen Grundlage setzt auch der extensiven Auslegung von Eingriffsnormen Grenzen. Therapeutische Beurteilungsspielräume im Maßregelvollzug ändern am Erfordernis einer gesetzlichen Eingriffsgrundlage nichts.

- „Grundrechte dürfen nur durch Gesetz oder aufgrund Gesetzes und nur unter Beachtung des Grundsatzes der Verhältnismäßigkeit eingeschränkt werden. Dies gilt allgemein und daher auch für Gefangene (vgl. BerfGE 33, $1<11>$; 89, $315<322 \mathrm{f}$. $>$; 116, $69<80>$; BVerfGK 2, $102<105>$ ) und im Maßregelvollzug Untergebrachte. Soweit eine sachgerechte Behandlung im Maßregelvollzug die Einräumung therapeutischer Beurteilungsspielräume erfordert, berührt dies

6 BVerfG II/2, Beschluss vom 30.10.2014 - 2 BvR 1513/14 -, juris, m.w.N.; als selbstverständlich vorausgesetzt auch in ständiger Sentasrechtsprechung, s. BVerfGE 35, 311 (315 f.); 57, 170 (177), Auszug weiter unten.

7 BVerfGE 58, 358 (367). 
nicht die grundsätzliche verfassungsrechtliche Notwendigkeit einer ausreichenden gesetzlichen Grundlage für Grundrechtsbeschränkungen im Maßregelvollzug (vgl. BVerfG, Beschluss der 2. Kammer des Zweiten Senats vom 12. November 2007 - 2 BvR 9/06 - www.bverfg.de). Damit sind auch einer erweiternden Auslegung bestehender Eingriffsnormen verfassungsrechtliche Grenzen gesetzt." ${ }^{\text {( }}$

Gesetzlicher Regelung bedarf auch das Resozialisierungskonzept, das dem Vollzug zugrundegelegt werden soll.

- „Das Erfordernis gesetzlicher Regelung betrifft über den Bereich der unmittelbar eingreifenden Maßnahmen hinaus auch die Ausrichtung des Vollzuges auf das Ziel der sozialen Integration (vgl. ...). Der Gesetzgeber selbst ist verpflichtet, ein wirksames Resozialisierungskonzept zu entwickeln und den Strafvollzug darauf aufzubauen (vgl. BVerfGE 98, $169<201>$ ). "9

Die Schließung von Regelungslücken im Wege der Analogie kommt, soweit nicht ohnehin bereichsbezogen ausgeschlossen, jedenfalls dann nicht in Betracht, wenn es an einer gesetzlichen Regelung nicht nur punktuell, sondern für ein ganzes grundrechtsrelevantes Sachgebiet fehlt.

\,Unabhängig davon ist fraglich, inwieweit außerhalb spezieller Analogieverbote, wie sie für das materielle Straf- und Disziplinarrecht und hinsichtlich der materiellrechtlichen Grundlagen von Freiheitsentziehungen (vgl. BVerfGE 29, 183 [195f.]; 34, 293 [301f.]; 83, 24 [31f.]) gelten, auch eine nur analog anwendbare gesetzliche Vorschrift dem Erfordernis einer gesetzlichen Grundlage für Grundrechtseingriffe genügen kann. Diese Frage bedarf hier keiner abschließenden Klärung. Nach dem Sinn und Zweck des Gesetzesvorbehalts scheidet eine Schließung von Regelungslücken im Wege der Analogie jedenfalls dann aus, wenn für eine ganze Rechtsmaterie mit vielfältigem Grundrechtsbezug der Gesetzgeber die Entscheidung über deren Ausgestaltung nicht getroffen und die dazu erforderlichen grundrechtsrelevanten Abwägungen nicht vorgenommen hat. So liegt es hier. Ausreichende

8 BVerfG II/2, Beschluss vom 12.3.2008 - 2 BvR 2219/06 -, juris, betr. $§ 9$ Abs. 2 MRVG NW.

9 BVerfGE 116, 69 (89). 
gesetzliche Eingriffsgrundlagen fehlen für beinahe den gesamten Bereich des Jugendstrafvollzuges. " ${ }^{10}$

Für den Bereich der Untersuchungshaft wurde $\S 119$ Abs. 3 StPO a.F. als ausreichende gesetzliche Grundlage angesehen.

- „Das Bundesverfassungsgericht hat keine Bedenken, daß § 119 Abs. 3 StPO eine verfassungsrechtlich zureichende gesetzliche Grundlage für Einschränkungen grundrechtlicher Freiheiten des Untersuchungsgefangenen bildet (BVerfGE 35, 311 [316]; st. Rspr.)."11

Verwaltungsvorschriften (VV) genügen als gesetzliche Grundlage selbstverständlich nicht; ihnen kommt keine normsetzende Bedeutung zu (BVerfG II/2, Beschluss vom 27.9.1995 - 2 BvR 903/95 u.a. -, juris). Allerdings kann, soweit Verwaltungsvorschriften zur Konkretisierung gesetzlicher Vorschriften erlassen sind, eine ausreichende gesetzliche Grundlage in der gesetzlichen Bestimmung zu sehen sein, zu deren Konkretisierung die Verwaltungsvorschrift ergangen ist (s. für die VV zu § 46 StVollzG BVerfG II/2, Beschluss vom 27.9.1995 - 2 BvR 903/95 u.a. -, juris). Entsprechendes gilt für die Hausordnung einer Justizvollzugsanstalt. Sie stellt grundsätzlich keine außenwirksame Rechtsnorm dar (BVerfGK 14, $381<385>$; s. allerdings dazu, dass Bestimmungen der Hausordnung unter besonderen Voraussetzungen als Allgemeinverfügung angreifbar sein können, BVerfG II/3, Beschluss vom 5.5.2011 - 2 BvR 722/11 -, juris) und ist daher auch nicht geeignet, selbständig disziplinarisch sanktionierbare Verhaltenspflichten zu begründen, die über die sich aus dem Gesetz ergebenden hinausgehen (BVerfG II/2, Beschluss vom 10.11.1995 - 2 BvR 1236/95 -, juris; BVerfG II/2, Beschluß vom 11.8.1997 - 2 BvR 2334/96 -, juris), kann aber sachgerechte Konkretisierungen gesetzlicher Verhaltenspflichten des Gefangenen enthalten (näher im Abschnitt zu Disziplinarmaßnahmen, unten B.XXVII.1.).

Auf Leistungen zur Sicherung des Existenzminimums muss ein Rechtsanspruch bestehen (BVerfGK 14, $99<106>$, m.w.N.). Ob dies bedeutet, dass der Anspruch nicht nur dem Grunde, sondern auch der Höhe nach gesetzlich festgelegt sein müssen, hat das Bundesverfassungsgericht

10 BVerfGE 116, 69 (83).

11 BVerfGE 57, 170 (177); dazu und zur aus der fehlenden Spezifität der Regelung folgende verfassungsrechtlichen Notwendigkeit restriktiver Auslegung näher unten im Kapitel zur Untersuchungshaft, D.I.2.a). 
in einem den Taschengeldanspruch von Maßregelvollzugspatienten betreffenden Fall offengelassen (BVerfGK 14, $99<106>$; s. allerdings zwischenzeitlich - nicht zum Strafvollzug, sondern zu Hartz-IV-Regelleistungen bzw. existenzsichernden Leistungen für Asylbewerber - BVerfGE 125, $175<223$ f. >; BVerfGE 132, $134<160$, Rn. 66>).

Wegen Fehlens einer gesetzlichen Grundlage hat das Bundesverfassungsgericht einen Verstoß gegen das Grundrecht aus Art. 2 Abs. 1 GG in der Aufrechnung von Kosten des gerichtlichen Verfahrens nach dem Strafvollzugsgesetz mit dem Taschengeldanspruch des Gefangenen gesehen (BVerfG II/2, Beschluss vom 14.8.1996 - 2 BvR 2088/93 -, juris, s. Auszug im nachfolgenden Abschnitt A.III.). Wegen unzureichender Prüfung des Vorhandenseins einer gesetzlichen Grundlage beanstandet wurden beispielsweise gerichtliche Bestätigungen der Rechtmäßigkeit eines zur Ahndung von Fehlverhalten verhängten Zimmerarrests im Maßregelvollzug (BVerfGK 12, $402<407 \mathrm{ff}$.>; Hinweis auf das Problem einer Rechtsgrundlage für eine mit Sanktionsfunktion erfolgte Entziehung von Gegenständen im Maßregelvollzug auch in BVerfGK 9, $460<465>$ ) und einer Verknüpfung der Besuchserlaubnis im Maßregelvollzug mit der Bedingung eines „Erstgesprächs“ zwischen Besucher und Therapeut (BVerfG II/2, Beschluss vom 12.3.2008 - 2 BvR 2219/06 -, juris).

Dagegen wurde angenommen, dass für die Nutzung der in $\S 24$ Abs. 2 EGGVG gesetzlich vorgesehenen Möglichkeit, dem gerichtlichen Verfahren nach $\S \S 23 \mathrm{ff}$. EGGVG auf Landesebene ein Vorverfahren vorzuschalten, eine bloße Verwaltungsvorschrift ausreicht (BVerfGE 40, 237 $<247$ f. $>$, s. Auszug im Abschnitt zum Verfahren nach $\S \S 23$ ff. EGGVG, unten E.III.5.)

\section{Normenbestimmtheit und -klarheit}

Gesetzliche Regelungen, insbesondere solche, auf deren Grundlage in Grundrechte eingegriffen wird, müssen ausreichend klar und bestimmt sein. Das schließt die Anforderung der Widerspruchsfreiheit ein. Der Grad der notwendigen Bestimmtheit lässt sich nicht allgemein bestimmen, sondern hängt von den Umständen, insbesondere vom Grad sinnvoller Bestimmbarkeit im Voraus, vom Gewicht des normierten Grundrechtseingriffs und von den Verständnismöglichkeiten und dem Orientierungsbedarf des jeweiligen Kreises der Normadressaten ab. 
- „Die Voraussetzungen für die Zulässigkeit des Eingriffs müssen hinreichend klar und bestimmt geregelt sein (vgl. für den Strafvollzug i.w.S. BVerfGE 116, $69<80>$, m.w.N.). Der Gesetzgeber ist gehalten, seine Vorschriften so bestimmt zu fassen, wie dies nach der Eigenart der zu ordnenden Lebenssachverhalte mit Rücksicht auf den Normzweck möglich ist (vgl. BVerfGE 49, $168<181>$; 59, $104<114>$; 78, 205 $<212>$; 103, $332<384>$ ). Die notwendige Bestimmtheit fehlt nicht schon deshalb, weil eine Norm auslegungsbedürftig ist (vgl. BVerfGE 45, $400<420>$; 117, $71<111>$; stRspr). Die Betroffenen müssen jedoch die Rechtslage erkennen und ihr Verhalten danach einrichten können (vgl. BVerfGE 103, $332<384>$; 113, $348<375>$, m.w.N.), und die gesetzesausführende Verwaltung muss für ihr Verhalten steuernde und begrenzende Handlungsmaßstäbe vorfinden (vgl. BVerfGE 110, 33 $<54>$; 113, // $348<375>$ ). Zur notwendigen Erkennbarkeit des Norminhalts gehört die Klarheit (vgl. BVerfGE 78, $214<226>$; 115, 166 $<190>$; 119, $331<366>$; stRspr) und, als deren Bestandteil, die Widerspruchsfreiheit (vgl. BVerfGE 98, $106<118$ f. >; 108, $169<181,183>$; 119, $331<366>$; stRspr) der Norm. Die Anforderungen an den Grad der Klarheit und Bestimmtheit sind umso strenger, je intensiver der Grundrechtseingriff ist, den eine Norm vorsieht (vgl. BVerfGE 59, 104 $<114>$; 75, $329<342>$; 86, $288<311>$; 110, $33<55>$; 117, $71<111>$ ). Für die näheren Anforderungen kann, nicht zuletzt in der Frage, inwieweit Maßgaben, die sich aus dem Grundgesetz ableiten lassen, ausdrücklicher und konkretisierender Festlegung im einfachen Gesetz bedürfen, auch der jeweilige Kreis der Normanwender und Normbetroffenen von Bedeutung sein (vgl. BVerfGE 110, $33<64>$; 123, 39 $<81>)$." 12

\section{Die Verwendung von Generalklauseln ist nicht ausgeschlossen.}

- „Die Grundrechte von Strafgefangenen können also nur durch oder aufgrund eines Gesetzes eingeschränkt werden, das allerdings auf möglichst engbegrenzte - Generalklauseln nicht wird verzichten können." 13

12 BVerfGE 128, 282 (317f.); zu Bestimmtheitsanforderungen s. auch BVerfG II/2, Beschluss vom 14.8.1996 - 2 BvR 2088/93 -, juris; BVerfGK 9, 460 (465); 12, 402 (407).

13 BVerfGE 33, 1 (11). 
Für unzureichend klar und bestimmt wurden verschiedene landesrechtliche Regelungen zur medizinischen Zwangsbehandlung von Untergebrachten befunden (näher C.II.1.).

\section{Strafvollzug und Internationale Standards}

Die Europäische Menschenrechtskonvention (EMRK) hat, wie auch andere internationale Verträge mit Menschenrechtsbezug, in Deutschland zwar keinen Verfassungsrang,

- „Innerhalb der deutschen Rechtsordnung stehen die Europäische Menschenrechtskonvention und ihre Zusatzprotokolle - soweit sie für die Bundesrepublik Deutschland in Kraft getreten sind - im Rang eines Bundesgesetzes (vgl. BVerfGE 74, 358 [370]; 82, 106 [120]; 111, 307 [316f.]). “14

sie dient aber, mit dem Inhalt, den die Rechtsprechung des Europäischen Gerichtshofs für Menschenrechte ihr zuspricht, als Auslegungshilfe für die verfassungsrechtlichen Gewährleistungen, die den menschenrechtlichen Gewährleistungen der EMRK korrespondieren.

- „Gleichwohl besitzen die Gewährleistungen der Europäischen Menschenrechtskonvention verfassungsrechtliche Bedeutung, indem sie die Auslegung der Grundrechte und rechtsstaatlichen Grundsätze des Grundgesetzes beeinflussen. Der Konventionstext und die Rechtsprechung des Europäischen Gerichtshofs für Menschenrechte dienen nach der ständigen Rechtsprechung des Bundesverfassungsgerichts auf der Ebene des Verfassungsrechts als Auslegungshilfen für die Bestimmung von Inhalt und Reich- // weite von Grundrechten und rechtsstaatlichen Grundsätzen des Grundgesetzes, sofern dies nicht zu einer - von der Konvention selbst nicht gewollten (vgl. Art. 53 EMRK) - Einschränkung oder Minderung des Grundrechtsschutzes nach dem Grundgesetz führt (vgl. BVerfGE 74, 358 [370]; 83, 119 [128]; 111, 307 [317]; 120, 180 [200 f.]; BVerfGK 3, 4 [7 f.]; 9, 174 [190 f.]; 10, 66 [77 f.]; 10, 234 [239]; 11, 153 [159 ff.]; 12, 37 [40]; BVerfG, Beschluss der 3. Kammer des Zweiten Senats vom 20. Dezember 2000 - 2 BvR 591/00 -, NJW

14 BVerfGE 128, 326 (367); die Entscheidung betrifft die Sicherungsverwahrung und deren erforderliche vollzugliche Ausgestaltung. 
2001, S. 2245 ff.; Beschluss der 2. Kammer des Ersten Senats vom 21. November 2002 - 1 BvR 1965/02 -, NJW 2003, S. 344 [345]; Beschluss der 3. Kammer des Ersten Senats vom 2. Juli 2008 - 1 BvR 3006/07 -, NJW 2008, S. 2978 [2981]; Beschluss der 2. Kammer des Ersten Senats vom 18. Dezember 2008 - 1 BvR 2604/06 -, NJW 2009, S. 1133 f.; Beschluss der 2. Kammer des Zweiten Senats vom 4. Februar 2010 - 2 BvR 2307/06 -, EuGRZ 2010, S. 145 [147]).

Im Rahmen der Heranziehung der Europäischen Menschenrechtskonvention als Auslegungshilfe berücksichtigt das Bundesverfassungsgericht Entscheidungen des Europäischen Gerichtshofs für Menschenrechte auch dann, wenn sie nicht denselben Streitgegenstand betreffen. Dies beruht auf der jedenfalls faktischen Orientierungs- und Leitfunktion, die der Rechtsprechung des Europäischen Gerichtshofs für Menschenrechte für die Auslegung der Europäischen Menschenrechtskonvention auch über den konkret entschiedenen Einzelfall hinaus zukommt (vgl. zur Orientierungswirkung der Rechtsprechung des Europäischen Gerichtshofs für Menschenrechte bereits BVerfGE 111, 307 [320]; BVerfGK 10, 66 [77 f.]; 10, 234 [239]; jeweils m. w. N.).“15

Das Grundgesetz ist nach Möglichkeit, d.h. im Rahmen des Vertretbaren und soweit damit keine Verringerung des Grundrechtsschutzes verbunden ist und die Verfassungsidentität gewahrt bleibt, so auszulegen, dass die Auslegung im Ergebnis - nicht notwendigerweise auch in den rechtsdogmatischen Strukturen und verwendeten Termini (zur Ergebnisorientierung s. BVerfGE 128, $326<370>$ ) - den Anforderungen der EMRK entspricht.

\, „Grenzen der völkerrechtsfreundlichen Auslegung ergeben sich aus dem Grundgesetz. Sie darf zunächst nicht dazu führen, dass der Grundrechtsschutz nach dem Grundgesetz eingeschränkt wird; das schließt auch die Europäische Menschenrechtskonvention selbst aus (vgl. Art. 53 EMRK, siehe BVerfGE 111, 307 [317] m. w. N.). Dieses Rezeptionshemmnis kann vor allem in mehrpoligen Grundrechtsverhältnissen relevant werden, in denen das „Mehr“ an Freiheit für den einen Grundrechtsträger zugleich ein „Weniger“ für einen anderen bedeutet (vgl. ...). Die Möglichkeiten einer konventionsfreundlichen Auslegung enden dort, wo diese nach den anerkannten Methoden der Gesetzesaus-

15 BVerfGE 128, 326 (367f.). 
legung und Verfassungsinterpretation nicht mehr vertretbar erscheint (vgl. BVerfGE 111, 307 [329]; ... zur absoluten Grenze des Kerngehalts der Verfassungsidentität des Grundgesetzes gemäß Art. 79 Abs. 3 GG vgl. BVerfGE 123, 267 [344]; ...).“16

Auch den Bestimmungen des Übereinkommens Nr. 29 der Internationalen Arbeitsorganisation kommt Bedeutung als Auslegungshilfe für das Grundgesetz zu.

- „Seit langem beanstanden Normen des internationalen Rechts eine voraussetzungslose Verdingung von Strafgefangenen. Art. 2 Abs. 2 lit.c) des ILO-Übereinkommens Nr. 29 vom 28. Juni 1930 (vgl. BGBl 1956 II S. 640; in der Bundesrepublik in Kraft seit 13. Juni 1957 [BGBl 1957 II S. 1694]) nimmt Zwangs- oder Pflichtarbeit von einem grundsätzlichen Verbot nur unter der Bedingung aus, daß diese „unter Überwachung und Aufsicht der öffentlichen Behörden ausgeführt wird...". Dieser schon bei der Beratung des Grundgesetzes vorzufindende internationale Standard liegt dem Willen des Verfassunggebers zugrunde und ist eine Auslegungshilfe auch für das Grundgesetz." 17

Eine indizielle Bedeutung enfalten internationale Standards mit Menschenrechtsbezug zudem auch dann, wenn es sich nicht um Normen des zwingenden Völkerrechts oder um völkervertragsrechtlich vereinbarte Standards handelt, sondern um sogenanntes soft law.

- „Auf eine den grundrechtlichen Anforderungen nicht genügende Berücksichtigung vorhandener Erkenntnisse oder auf eine den grundrechtlichen Anforderungen nicht entsprechende Gewichtung der Belange der Inhaftierten kann es hindeuten, wenn völkerrechtliche Vorgaben oder internationale Standards mit Menschenrechtsbezug, wie sie in den im Rahmen der Vereinten Nationen oder von Organen des Europarates beschlossenen einschlägigen Richtlinien und Empfehlungen enthalten sind (vgl. Höynck/Neubacher/Schüler-Springorum, Internationale Menschenrechtsstandards und das Jugendkriminalrecht. Dokumente der Vereinten Nationen und des Europarates, hg. vom Bundesministerium der Justiz in Zusammenarbeit mit der Deutschen Vereinigung für Jugendgerichte und Jugendgerichtshilfen e.V., 2001; Empfehlungen

16 BVerfGE 128, $326(371)$.

17 BVerfGE 98, 169 (206), s. auch BVerfG II/2, Beschluss vom 3.0.2011 - 2 BvR $176 / 11-$, juris. 
des Europarates zum Freiheitsentzug 1962 - 2003, hg. vom Bundesministerium der Justiz, Berlin, Bundesministerium für Justiz, Wien, und Eidgenössischen Justiz- und Polizeidepartement, Bern, 2004), nicht beachtet // beziehungsweise unterschritten werden (vgl. auch Schweizerisches Bundesgericht, Urteil vom 12. Februar 1992, BGE 118 Ia 64 $<70>$ )." 18

Dementsprechend wurden zum Beispiel die Europäischen Strafvollzugsgrundsätze und die UN-Mindestregeln für die Behandlung der Gefangenen herangezogen zur Klärung der grundrechtlichen Anforderungen an die Beschaffenheit von Hafträumen (BVerfGK 12, $422<424 \mathrm{ff} .>$ ). Die Nichtberücksichtigung der Europäischen Strafvollzugsgrundsätze und eines Berichts des Europäischen Komitees zur Verhütung von Folter und unmenschlicher oder erniedrigender Behandlung oder Strafe (CPT-Bericht) wurde gerügt in einem die Aufschlusszeiten für Untersuchungsgefangene betreffenden Fall (BVerfGK 20, $93<104>$ ).

\section{Grundrechtsmemorabilien}

Um Grundrechtsfragen geht es in sämtlichen auf Verfassungsbeschwerden hin ergangenen Sachentscheidungen des Bundesverfassungsgerichts. Die Inhalte dieser Rechtsprechung werden in den weiteren Hauptkapiteln dargestellt. Im vorliegenden Abschnitt sollen nur einige Grundrechtslehren in Erinnerung gerufen - beziehungsweise für die, die die sich mit Grundrechten bislang nicht näher beschäftigt haben, vorgestellt - werden, die teils in der vollzuglichen Praxis oder in der Praxis der Gerichte in Vollzugssachen, teils in der Argumentation von Gefangenen oder ihren Anwälten häufig übersehen werden.

\section{Voraussetzungen eingriffsausschließender Zustimmung}

Eine eingriffsausschließende Zustimmung zu einer vollzugsbehördlichen Maßnahme setzt voraus, dass der Betroffene einwilligungsfähig und keinem unzulässigen Zustimmungsdruck ausgesetzt ist.

18 BVerfGE 116, 69 (90 f.); s. auch BVerfGK 20, 93 (101). 
„Die Eingriffsqualität entfällt auch nicht bereits dann, wenn der Betroffene der abgelehnten Behandlung keinen physischen Widerstand entgegensetzt. Das bloße Aufgeben einer bestimmten Form des Protests kann nicht ohne Weiteres als Zustimmung ge- // deutet werden. Die medizinische Behandlung eines Untergebrachten, die ihrer Art nach das Grundrecht auf körperliche Unversehrtheit berührt, greift in dieses Grundrecht allenfalls dann nicht ein, wenn sie von der frei, auf der Grundlage der gebotenen ärztlichen Aufklärung, erteilten Einwilligung des Untergebrachten gedeckt ist. Dies setzt voraus, dass der Untergebrachte einwilligungsfähig ist (vgl. BGHZ 29, 46 [51]; 154, 205 [210]) und keinem unzulässigen Druck ausgesetzt wurde, etwa durch das Inaussichtstellen von Nachteilen im Falle der Behandlungsverweigerung, die sich nicht als notwendige Konsequenzen aus dem Zustand ergeben, in dem der Betroffene unbehandelt voraussichtlich verbleiben oder in den er aufgrund seiner Weigerung voraussichtlich geraten wird.“19

Eine frei erteilte Zustimmung zu einer Maßnahme der Briefüberwachung liegt daher nicht vor, wenn die Zustimmung zur Voraussetzung einer Aushändigung gemacht worden war, die von dieser Zustimmung nicht hätte abhängig gemacht werden dürfen. Bei der Beurteilung der Freiwilligkeit der Zustimmung zu einem Eingriff sind zudem die besonderen Abhängigkeitsverhältnisse im Vollzug zu berücksichtigen.

- „Die Einwilligung des Betroffenen kann den grundrechtseingreifenden Charakter einer behördlichen Maßnahme nur ausschließen, wenn sie frei erteilt wurde (vgl. BVerfG, Beschluss des Zweiten Senats vom 23. März 2011 - 2 BvR 882/09 -, NJW 2011, S. $2113<2114>$ ). Die Frage, inwieweit unter den besonderen, durch weitreichende Abhängigkeitsverhältnisse geprägten Bedingungen des Strafvollzuges von Freiwilligkeit die Rede sein kann, wenn ein Gefangener grundrechtseingreifenden Maßnahmen von Vollzugsbediensteten zustimmt, muss hier nicht allgemein beantwortet werden. Die Annahme einer frei erteilten, eingriffsausschließenden Einwilligung setzt jedenfalls voraus, dass die Zustimmung zu der grundrechtseingreifenden Maßnahme frei von unzulässigem Druck erfolgte (vgl. BVerfG, a.a.O., S. 2114; BVerfG, Beschluss des Vorprüfungsausschusses des Zweiten Senats

19 BVerfGE 128, 282 (301); s. auch nachfolgenden Beschlussauszug. 
vom 18. August 1981 - 2 BvR 166/81 -, NJW 1982, S. 375 $<375>$; ...).

Ein solcher Fall lag hier nicht vor. Das Oberlandesgericht selbst hat aufgrund der Feststellungen des Landgerichts angenommen, dass mit der erfolgten Rückfrage beim Absender des Briefes, um den es hier geht, dessen Eigenschaft als Verteidigerpost geklärt war und der Brief daher gemäß $§ 24$ Abs. 2 Satz 1 JVollzGB III BW nicht der Überwachung unterlag, mit der Folge grundsätzlicher Unzulässigkeit einer Öffnung des Schreibens. Nach dieser Feststellung war die Anstalt auch nicht berechtigt, dem Beschwerdeführer das Schreiben vorzuenthalten, sondern verpflichtet, es ihm ungeöffnet auszuhändigen, und daher jedenfalls aus diesem Grund auch nicht berechtigt, die Aushändigung davon abhängig zu machen, dass der Beschwerdeführer sich mit einer Sichtkontrolle einverstanden erklärte." ${ }^{\prime 20}$

Wird ein Gefangener oder Untergebrachter vor die Wahl zwischen zwei Alternativen gestellt, so entfällt der eingreifende Charakter jeder der Alternativen nicht deshalb, weil der Betroffene jeder von ihnen ausweichen kann, indem er sich für die jeweils andere entscheidet. Daher kann zum Beispiel eine grundrechtsrelevante Belastung durch die Versagung von Vollzugslockerungen nicht mit der Begründung verneint werden, dass der Betroffene sich die Möglichkeit solcher Lockerungen durch Zustimmung zu seiner Verlegung in eine andere Vollzugseinrichtung verschaffen könne.

- „Für das auf Ausgänge aus dem Bezirkskrankenhaus Straubing gerichtete Klagebegehren entfiel das Rechtsschutzbedürfnis nicht dadurch, dass dem Beschwerdeführer Ausgänge aus dem Bezirkskrankenhaus Haar für den Fall seiner Zustimmung zur Verlegung dorthin in Aussicht gestellt worden waren. Weder war damit seinem Begehren schon Rechnung getragen, noch ist der Unterschied zwischen dem, was der Beschwerdeführer begehrte - Ausgänge aus der Straubinger Klinik -, und dem, was ihm in Aussicht // gestellt worden war - eventuelle Gewährung von Ausgängen nach einer Verlegung -, in rechtlicher Hinsicht derart offensichtlich irrelevant, dass es angehen könnte, schon das Interesse an der Klärung der Berechtigung des weitergehenden Begehrens zu verneinen. $\mathrm{Ob}$ der Beschwerdeführer sich, um begehrte Voll-

20 BVerfGK 19, 140 (147). 
zugslockerungen erreichen zu können, zunächst auf die Verlegung in eine andere Klinik einlassen muss, ist im Gegenteil eine Frage, die seine Rechtssphäre in erheblicher Weise berührt (vgl. zur Grundrechtsrelevanz ungewollter Verlegungen BVerfGK 6, $260<264>$ m.w.N.). Wird eine die Rechtssphäre des Antragstellers berührende behördliche Entscheidung, wie im vorliegenden Fall die Entscheidung über Vollzugslockerungen, an Bedingungen - hier insbesondere die Bedingung einer vorgängigen Verlegung - geknüpft, die ihrerseits die Rechtssphäre des Antragstellers berühren oder möglicherweise berühren, dann kann weder das Interesse des Antragstellers an gerichtlichem Rechtsschutz gegen diese Verknüpfung verneint noch der gebotenen Sachprüfung dadurch ausgewichen werden, dass das Begehren des Antragstellers so behandelt wird, als beziehe es sich nicht auf die Verknüpfung, sondern nur auf eines ihrer Elemente.“21

Eine wirksame Einwilligung in menschenwürdewidrige Behandlung scheidet möglicherweise von vornherein aus. Das Bundesverfassungsgericht hat hierzu bislang keine Entscheidung getroffen, in einem Kammerbeschluss aber mit dem Verweis auf einschlägige fachgerichtliche Rechtsprechung deutliche Bedenken zum Ausdruck gebracht.

- „Deutet man die Ausführungen des Landgerichts dahin, dass es zum Ausdruck habe bringen wollen, der Beschwerdeführer habe konkludent in die räumlichen Haftbedingungen eingewilligt, sei mithin aufgrund eines Grundrechtsverzichts nicht in seiner Menschenwürde verletzt worden, ist dies ebenfalls nicht tragfähig. Zum einen hat das Landgericht nicht geprüft, ob die Annahme einer konkludenten Einwilligung des Beschwerdeführers in seiner Eigenschaft als Untersuchungsgefangener schon einfachrechtlich durch $\S 119$ Abs. 2 Satz 1 StPO a.F. (in der für das Verfahren maßgeblichen Fassung vom 7. April 1987, gültig bis zum 31. Dezember 2009) verstellt war beziehungsweise ob die von der Rechtsprechung entwickelten Anforderungen an eine konkludente Einwilligung des Beschwerdeführers in seiner Eigenschaft als Strafgefangener erfüllt waren (vgl. ...). Zum anderen hat das Landgericht nicht geprüft, ob die Menschenwürde überhaupt ein disponibles Grundrecht ist, das einen Grundrechtsverzicht zulässt (vgl. ablehnend: BVerwG, Urteil vom 17. Oktober 2000 - BVerwG 2 WD 12/00, 13/00

21 BVerfGK 11, 262 (266f). 
-, NJW 2001 S. $2343<2344>$; BSG, Urteil vom 6. Mai 2009 - B 11 AL 11/08 -, juris Rn. 25). Auch diese Rechtsfragen können nicht als hinreichend - zu Lasten des Beschwerdeführers - geklärt im Sinne des oben erörterten Maßstabes der Rechtsschutzgleichheit für die Bewilligung von Prozesskostenhilfe angesehen werden. “22

Es liegt kein Pflichtverstoß darin, wenn ein Gefangener eine eingriffsausschließende Zustimmung zu einer behördlichen Maßnahme verweigert. Die Gehorsamspflicht des Gefangenen schließt keine Zustimmungspflicht in dem Sinne ein, dass dem Gefangenen abverlangt werden könnte, sich angeordneten Maßnahmen unter Verzicht auf deren gerichtliche Überprüfung zu unterwerfen.

- „Der Beschwerdeführer hatte geltend gemacht, er habe seine Zustimmung zu der Verlegung verweigert. Sollte seine Weigerung nicht weiter als bis hierher gegangen sein, könnte darin ein Pflichtverstoß von vornherein nicht gesehen werden, denn auch soweit der Gefangene verpflichtet ist, Anweisungen der Justizvollzugsanstalt Folge zu leisten, ist er nicht verpflichtet, ihnen im Sinne der Herstellung eines Einvernehmens "zuzustimmen" und sich damit der Möglichkeit zu begeben, die Anweisung gerichtlich überprüfen zu lassen. Es ist vielmehr Sache der Strafvollstreckungskammer, Gefangene davor zu schützen, dass eine Justizvollzugsanstalt Gefangene mit Nachteilen wegen der Verweigerung einer solchen Zustimmung überzieht und auf diese Weise Druck in Richtung auf einen Verzicht auf das Geltendmachen von Rechten ausübt." 23

\section{Rechtsstaatliche Zurechnung}

Bei grundrechtseingreifenden Maßnahmen müssen die Grundsätze rechtsstaatlicher Zurechnung berücksichtigt werden. Rechtswidriges Verhalten darf nicht dadurch begünstigt werden, dass seine Urheber damit rechnen können, eingreifende staatliche Reaktionen darauf würden Dritte treffen. Gefahrenabwehrende Maßnahmen sind danach vorrangig gegen die für die Entstehung der Gefahr Verantwortlichen zu richten.

22 BVerfG I/1, Beschluss vom 22.2.2011 - 1 BvR 409/09 -, juris.

23 BVerfG II/3, Beschluss vom 20.3.2013 - 2 BvR 2941/12 -, juris. 
„Es war und ist daher Sache des Beschwerdeführers, die angegebene Veränderung der Sachlage zunächst mit einem Antrag auf Aufhebung der ihn belastenden Maßnahmen vor dem zuständigen Gericht geltend zu machen.

Dieses wird bei seiner Entscheidung auch die Grundsätze rechtsstaatlicher Zurechnung zu berücksichtigen haben. Mit diesen Grundsätzen ist es unvereinbar, wenn die Gefahr, dass bestimmte Personen sich in rechtswidriger Weise verhalten, nicht im Regelfall vorrangig diesen Personen zugerechnet und nach Möglichkeit durch ihnen gegenüber zu ergreifende Maßnahmen abgewehrt wird, sondern ohne weiteres Dritte oder gar die potentiellen Opfer des drohenden rechtswidrigen Verhaltens zum Objekt eingreifender Maßnahmen der Gefahrenabwehr gemacht werden (vgl. BVerfGE 69, $315<360 \mathrm{f}$.>; BVerfGK 6, 260 $<265>$; 8, $307<311>$ ). Rechtsstaatliche Zurechnung muss darauf ausgerichtet sein, nicht rechtswidriges, sondern rechtmäßiges Verhalten zu begünstigen (vgl. BVerfGE 116, $24<49>$ ). Dem läuft es grundsätzlich zuwider, wenn Maßnahmen zur Abwehr drohenden rechtswidrigen Verhaltens nicht vorrangig gegen den oder die Störer, sondern ohne weiteres - und in Grundrechte eingreifend - gegen den von solchem rechtswidrigen Verhalten potentiell Betroffenen ergriffen werden (BVerfGK 8, $307<311>$ ). Sind in einer Haftanstalt Maßnahmen zum Schutz eines Gefangenen vor Bedrohung durch Dritte erforderlich, müssen daher vorrangig bestehende - gegebenenfalls auch disziplinarische - Möglichkeiten der Einwirkung auf diejenigen ausgeschöpft werden, von denen die Bedrohung ausgeht. Eingreifende Maßnahmen gegenüber dem Bedrohten dürfen die Gerichte nicht anordnen oder billigen, ohne geprüft zu haben, ob sie nach diesen Grundsätzen unentbehrlich sind." 24

\section{Aspekte des Grundsatzes der Verhältnismäßigkeit}

Dass für den Haftvollzug im Ganzen wie für die einzelnen dabei anfallenden Eingriffe in Grundrechte der Gefangenen der Grundsatz der Verhält-

24 BVerfG II/3, Beschluss vom 22.7.2010 - 2 BvR 1528/10 -, juris, betr. gegen einen Gefangenen zu seinem Schutz vor Angriffen Mitgefangener verhängte Sicherungsmaßnahmen; zur Parallelkonstellation der Verlegung eines Gefangenen zu seinem Schutz BVerfGK 8, 307 (311), Auszug unter B.VIII.1. 
nismäßigkeit gilt, ist allgemein bekannt. Die Folgerungen, die sich hieraus im Einzelnen ergeben, hier zusammenfassend darzustellen, würde nur zu unnötigen Verdoppelungen im Verhältnis zu den nachfolgenden Kapiteln führen. Dem Zweck des vorgezogenen Abschnitts zu einigen Grundrechtsfragen von übergreifender Bedeutung entsprechend (s.o. vor 1.) soll an dieser Stelle nur auf einige Gesichtspunkte hingewiesen werden, die offenbar weniger allgemein bekannt sind.

Häufig wird übersehen, dass zur Verhältnismäßigkeit eines Eingriffs die Erforderlichkeit gehört, an der es fehlt, wenn ein milderes Mittel gleichermaßen zwecktauglich wäre, und dass Entscheidungen, die in Grundrechte eines Gefangenen eingreifen, der Aufhebung wegen mangelnder Beachtung des Grundsatzes der Verhältnismäßigkeit schon dann unterliegen, wenn eine naheliegende Prüfung, ob der Einsatz eines milderen Mittels ausgereicht hätte, unterblieben ist - so zum Beispiel, wenn nicht geprüft wurde, ob die mit der Weiterleitung eines Schriftstücks verbundenen Gefahren statt durch vollständiges Anhalten auch durch bloße Schwärzung von Passagen ausgeräumt werden können (s. BVerfGK 4, $305<312>$, Auszug unter B.XIV.2.a), oder ob Gefahren, die sich wegen befürchteter Angriffe seitens Mitgefangener oder aus unzureichender Beaufsichtigung eines Gefangenen ergeben, statt durch Verlegung des Gefangenen durch Einwirkung auf die die Mitgefangenen (BVerfGK 8, $307<311$ f. $>$ ) oder die zuständigen Bediensteten begegnet werden könnte.

- „Die Auslegung und Anwendung des $\S 85$ StVollzG durch das Landgericht ist zudem unverhältnismäßig. Beschränkungen des Grundrechts aus Art. 2 Abs. 1 GG dürfen nur unter Beachtung des Verhältnismäßigkeitsgrundsatzes // vorgenommen werden; sie müssen zum Schutz eines kollidierenden Rechtsguts geeignet und erforderlich sein und zur Art und Intensität der Beeinträchtigung oder Gefährdung, der begegnet werden soll, in einem angemessenen Verhältnis stehen (vgl. BVerfGE 16, $194<201$ f. $>$; 92, $277<327$ f. >; stRspr). Unter der vom Landgericht angenommenen Voraussetzung, dass das Nichteinschreiten der Stationsbediensteten eine Gefahr im Sinne des $\S 85$ StVollzG darstellte, wäre zunächst festzustellen gewesen, ob der unterstellten Gefahr durch eine geeignete Einwirkung auf die Bediensteten, beispielsweise durch die schlichte Anweisung, Pflichtverstöße des Beschwerdeführers den Vorgesetzten zu melden, als milderes Mittel hätte begegnet werden 
können. Schon an dieser die Erforderlichkeit der Verlegung betreffenden Prüfung fehlt es." 25

Die Erforderlichkeit ist auch dann nicht gewahrt, wenn der Besitz von Geräten wegen Gefährdungen versagt wird, denen ausreichend durch weniger eingreifende Sicherungsmaßnahmen wie z.B. eine Verplombung begegnet werden könnte (BVerfG II/2, Beschluss vom 31.3.2003 - 2 BvR 1848/02 - juris; w.N. im Abschnitt zum Besitz von Gegenständen, B.XXI. 1.)

$\mathrm{Da}$ es für die Beurteilung der Verhältnismäßigkeit eines Eingriffs überwiegend auf Objektives ankommt, wird leicht übersehen, dass für die Beantwortung der Frage, welche von mehreren denkbaren Beschränkungen oder Beeinträchtigungen das mildere Mittel darstellt, die Wahrnehmung des betroffenen Inhaftierten zu berücksichtigen ist.

- „Die angegriffene Entscheidung prüft auch nicht, ob etwaigen aus Besuchskontakten hervorgehenden Gefahren für die Sicherheit und das geordnete Zusammenleben in der Klinik auf andere - weniger und gezielter eingreifende - Weise als durch verordnete Erstgespräche noch rechtzeitig und hinreichend wirksam begegnet werden könnte. Unter anderem lässt sie jede Auseinandersetzung mit der vom Beschwerdeführer bereits im fachgerichtlichen Verfahren aufgeworfenen Frage vermissen, ob nicht eine Besuchsüberwachung als milderes, gleich geeignetes Mittel anzusehen wäre. Die Überwachung von Besuchen stellt zwar gegenüber dem Abhängigmachen der Besuchsgenehmigung von einem zuvor absolvierten Erstgespräch mit den künftigen Besuchern nicht grundsätzlich das mildere Mittel dar. Bei unfreiwilliger Zufügung könnte durchaus die Überwachung als eingriffsintensiver empfunden werden. Beurteilt jedoch der Untergebrachte selbst dies anders, so ist dies, soweit es um seine grundrechtlich geschützte Sphäre geht, für die Feststellung der Schwere des Eingriffs zu berücksichtigen (vgl. BVerfGE $89,315<324>$ ). “26

„Das Trennungsgebot ist kein Selbstzweck, sondern dient der Besserstellung der Sicherungsverwahrten (vgl. BVerfGE 128, $326<380>$ ).

25 BVerfGK 6, 260 (265 f.); zum Gesichtspunkt rechtsstaatlicher Zurechnung in diesem Zusammenhang s. o. A.V.2.

26 BVerfG, Beschluss der 2. Kammer des Zweiten Senats vom 12.3.2008 - 2 BvR 2219/06 -, juris. 
Der Beschwerdeführer hatte substantiiert geltend gemacht, dass die Verlegung auf die Station für Sicherungsverwahrte wegen der dortigen Haftraumbedingungen und sonstiger baulicher Gegebenheiten, der personellen Ausstattung sowie des Freizeitangebots objektiv und nach seinen persönlichen Bedürfnissen (zur Bedeutung des subjektiven Empfindens für die grundrechtliche Beurteilung der Schwere einer Beeinträchtigung vgl. BVerfGE 89, $315<322 \mathrm{f}$. $>$ ) eine erhebliche Verschlechterung der Vollzugsbedingungen darstelle. Angesichts dieses von der Justizvollzugsanstalt in tatsächlicher Hinsicht nicht in Abrede gestellten - hinsichtlich der Haftraumbedingungen vielmehr mit dem Hinweis auf beabsichtigte bauliche Veränderungen ausdrücklich bestätigten - Vorbringens verkehrt die Rechtfertigung der Verlegung unter Verweis auf das Gebot der getrennten Unterbringung von Sicherungsverwahrten und Strafgefangenen den Sinn des Trennungsgebotes in sein Gegenteil.“27

Viele im Vollzug aus Gründen der Sicherheit oder Ordnung übliche Beschränkungen - unter anderem beispielsweise Lockerungsversagungen und Beschränkungen des Besitzes von Gegenständen - wären theoretisch vermeidbar, wenn höherer Aufwand, insbesonderer höherer personeller Überwachungsaufwand, betrieben würde, um der Gefahr, um die es jeweils geht, entgegenzuwirken. Gefangene ziehen daraus häufig den Schluss, dass die behauptete Gefahr gar nicht bestehe oder die betreffende Beschränkung unverhältnismäßig, da nicht erforderlich sei. Das trifft jedoch nicht ohne Weiteres zu, da kein grundrechtlicher Anspruch darauf besteht, dass zur Eingriffsvermeidung beliebig hohe Mittel eingesetzt werden (näher dazu weiter unten im Abschnitt über das Problem der knappen Ressourcen im Vollzug, A.VI.). Im Sinne des Grundsatzes der Verhältnismäßigkeit erforderlich, um einer Gefahr zu begegnen, ist eine Beschränkung daher nicht erst dann, wenn es überhaupt keine Möglichkeit gibt, der Gefahr auf eine den Inhaftierten weniger belastende Weise entgegenzuwirken, sondern schon dann, wenn dies nicht mit angemessenem Aufwand möglich ist. Diesen Zusammenhang zwischen der Angemessenheit des Aufwandes, insbesondere des Kontrollaufwandes, der zur Gefahrenvermeidung nötig wäre, und der Rechtmäßigkeit von Beschränkungen aus Sicherheitsgründen haben umgekehrt auch die Justizvollzugsanstalten zu beachten. Mit einer Gefahr für Sicherheit und Ordnung können sie Be- 
schränkungen nur rechtfertigen, wenn der Gefahr nicht mit angemessenem Aufwand auf eine für den Gefangenen weniger belastende Weise ausreichend begegnet werden kann (s. statt vieler BVerfGK 17, $429<432>$; Näheres im Abschnitt zum Besitz von Gegenständen im Strafvollzug, B.XXI. 1.; dort auch zum insoweit berücksichtigungsfähigen Gesichtspunkt der Verallgemeinerbarkeit für alle gleichartigen Fallgestaltungen).

\section{Gleichbehandlung}

Gefangene und Untergebrachte beschweren sich häufig über einen Gleichheitsverstoß, der darin liege, dass Gesetzesinhalte oder Vollzugspraxis in dem Bundesland, in dem sie inhaftiert sind, von derjenigen in einem anderen Bundesland zu ihrem Nachteil abweichen. Dem liegt ein Missverständnis zugrunde. Wollte man annehmen, der Gleichheitsgrundsatz des Art. 3 Abs. 1 GG verpflichte Landesgesetzgeber und Landesverwaltungen zu einheitlicher Nutzung der Spielräume, die ihnen nach der föderalen Ordnung des Grundgesetzes zustehen, so wäre diese föderale Ordnung hinfällig. Der Gleichheitsgrundsatz gilt nur im Rahmen der bundesstaatlichen Kompetenzordnung.

- „Durch Unterschiede in der Vollstreckungsplanung und Vollstreckungspraxis, die sich daraus ergeben, dass verschiedene Länder bestehende Beurteilungs- und Ermessensspielräume in unterschiedlicher Weise ausfüllen, wird der Beschwerdeführer auch nicht in seinem Grundrecht aus Art. 3 Abs. 1 GG verletzt. Dieses Grundrecht gilt nur im Rahmen der bundesstaatlichen Kompetenzordnung; es gewährleistet daher nicht, dass die Länder ihre Gesetzgebungskompetenzen sowie die Spielräume, die das einfache Bundesrecht ihnen für ihre Verwaltungstätigkeit einräumt, inhaltsgleich ausfüllen (vgl. BVerfGE 10, 354 $<371>$; $21,54<68>$; 76, $1<73>$ ). “28

Auch innerhalb ein und desselben Bundeslandes können Unterschiede in der Gesetzesanwendung durch verschiedene Anstalten gerechtfertigt sein. Insbesondere liegt ein Gleichheitsverstoß nicht vor, wenn unterschiedliche Vorgehensweisen in sachlich nachvollziehbarer Weise an Unterschiede in den tatsächlichen Verhältnissen der Anstalt anknüpfen (s. für Unter-

28 BVerfGK 12, 210 (223); s. auch BVerfG, Beschluss vom 22.8.1980 - 2 BvR 930/80 - Die Justiz 1980, 489 (in juris nur Orientierungssatz). 
schiede in der Ausstattung der Anstalt BVerfGK 14, $381<386>$; speziell für unterschiedliche Besuchszeiten BVerfG II/2, Beschluss vom 13.8.1993 - 2 BvR 1469/93 -, juris; s. allerdings dazu, dass unter Umständen Ausstattungsunterschiede ihrerseits $\mathrm{zu}$ problematisieren sein können, BVerfGK 14, $381<386$ f.>, Auszug im Abschnitt zur telefonischen Kommunikation im Strafvollzug, B.XV.; vgl. auch - nicht unter Gleichheitsgesichtspunkten thematisiert, aber offensichtlich auch unter diesem Gesichtspunkt relevant -, zur Sicherheitsstufe, in die eine Anstalt eingeordnet ist, als rechtfertigendem Gesichtspunkt für bestimmte Maßnahmen der Postkontrolle BVerfGK 2, $78<79$ f. $>$ ).

Ergänzend ist hinzuzufügen: Wegen der Unabhängigkeit der Gerichte folgt aus Art. 3 Abs. 1 GG auch kein Anspruch darauf, dass unterschiedliche Gerichte ein und dieselbe Regelung gleich auslegen (s. jeweils nicht zum Strafvollzug, aber selbstverständlich auch hier geltend, BVerfGE 87, $273<278>$; 126, $369<395>$ ). Ein Grundrechtsverstoß kann sich bei Abweichung eines Gerichts von der Rechtsprechung anderer Gerichte allerdings aus der Verletzung einer für solche Fälle vorgesehenen Vorlagepflicht ergeben (dazu unter E.II.17.).

Soweit es um eine - an Art. 3 Abs. 3 Satz 1 GG zu messende - unterschiedliche Behandlung männlicher und weiblicher Gefangener geht (dazu BVerfGK 14, $381<386$ f.>, Auszug im Abschnitt zur telefonischen Kommunikation im Strafvollzug, B.XV.), kann leicht aus dem Blick geraten, dass auch eine mittelbare Diskriminierung - d.h. eine gleichheitswidrige Benachteiligung, die ohne direkte Anknüpfung an das Geschlecht erreicht wird - zu vermeiden ist. Eine verbotene mittelbare Diskriminierung liegt allerdings nicht vor, wenn sich eine ausschließlich an Gesichtspunkte der Sicherheit anknüpfende Praxis auf männliche und weibliche Gefangene unterschiedlich auswirkt.

- „Das Gericht hat es jedoch versäumt, die gegebene Begründung daraufhin zu befragen, ob sie auch und gerade im Hinblick auf die praktizierten Unterschiede in der Behandlung der männlichen Gefangenen und der weiblichen Gefangenen tragfähig war.

Eine insoweit tragfähige Rechtfertigung ist nicht von vornherein ausgeschlossen. So ist denkbar, dass konkrete Erfahrungen und objektivierbare Anhaltspunkte wie zum Beispiel Deliktsstruktur, Art und Häufigkeit disziplinarisch $\mathrm{zu}$ ahndenden Fehlverhaltens, vorgekommene Fluchtversuche oder Lockerungsmissbräuche, Anzahl der Drogenfunde u.ä. die Annahme rechtfertigen, dass von den Insassen bestimmter 
Hafthäuser oder Abteilungen einer Anstalt im Ganzen so deutlich geringere Gefahren für die Sicherheit der Anstalt ausgehen, dass in der betreffenden Einheit, anders als in anderen, auf eine systematische Überwachung von Telefongesprächen im Allgemeinen verzichtet und infolgedessen eine weitergehende Telefonnut- // zung gestattet werden kann. Eine unterschiedliche Behandlung, die in dieser Weise an Sicherheitsgründe anknüpfte, wäre auch dann verfassungsrechtlich nicht zu beanstanden, wenn sich erweisen sollte, dass sie im Ergebnis faktisch zu einer Besserstellung gerade der inhaftierten Frauen führt. Denn diese Besserstellung ergäbe sich nicht aufgrund einer Anknüpfung an das Geschlecht, sondern nur als Folge des Abstellens auf die Vollzugssicherheit. Diese ist als notwendiges Element eines funktionsfähigen Strafvollzuges ein Belang von verfassungsrechtlichem Gewicht (vgl. BVerfGE 33, $1<13$ f.>; 89, $315<323>$; 116, $69<92$ f.>) und daher geeignet, die mittelbaren Folgen, die sich aus einem sachgerecht an ihr ausgerichteten Vorgehen ergeben, auch vor Art. 3 Abs. 3 Satz 1 GG zu rechtfertigen (vgl. BVerfGE 113, $1<20>$ ). “29

\section{Das Problem der knappen Ressourcen im Vollzug}

Verfassungsrechtliche Vorgaben für den Justizvollzug haben Kosten. Der verfassungsrechtlich geforderte resozialisierungsorientierte Strafvollzug (s. u. B.I.) zum Beispiel, die Wahrung des Grundsatzes der Verhältnismäßigkeit bei Grundrechtseingriffen, ja sogar die Wahrung der Menschenwürde von Inhaftierten erfordern den Einsatz personeller und sonstiger Mittel. Therapeutische Angebote, begleitete Vollzugslockerungen, Betreuung durch Sozialarbeiter, Schutz der Gefangenen vor wechselseitiger Gewalt, menschenwürdige Haftraumverhältnisse - all das gibt es nicht umsonst. Selbst die Einräumung von Betätigungs- und Kontaktmöglichkeiten aller Art, die auf den ersten Blick als „kostenlos“ erscheinen mögen, ist wegen damit aus Sicherheitsgründen einhergehender Aufsichts- und sonstiger Kontrollerfordernisse, beispielsweise zur Verhinderung des Einschmuggelns und Versteckens von Drogen und Ausbruchswerkzeugen, typischerweise mit erheblichem Personalaufwand verbunden. Dieser personelle Aufwand wird von Außenstehenden leicht unterschätzt, weil man 
sich keine Vorstellung von der außerordentlichen Kreativität macht, die viele Inhaftierte entfalten, um sich den auferlegten Beschränkungen zu entziehen.

Die verfassungsrechtlichen Anforderungen an die Ausgestaltung des Strafvollzuges schließen die Pflicht des Staates zur Bereitstellung der erforderlichen Mittel ein. Dies hat das Bundesverfassungsgericht schon im Zusammenhang mit der Forderung, den Strafvollzug auf eine gesetzliche Grundlage zu stellen, ausgesprochen und zugleich durch Verweis auf den „Rahmen des Zumutbaren“ verdeutlicht, dass die Reichweite der verfassungsrechtlichen Anforderungen ihrerseits nicht ohne Berücksichtigung der Kosten bestimmt werden kann.

- „Finanzielle Erwägungen oder organisatorische Schwierigkeiten, die ein Strafvollzugsgesetz mit sich bringen mag, dürfen eine Verabschiedung nicht unangemessen verzögern. Vielmehr muß der Staat den Strafvollzug so ausstatten, wie es zur Realisierung des Vollzugsziels (BVerfGE 35, 202 [235]) erforderlich ist. Es ist seine Aufgabe, im Rahmen des Zumutbaren alle gesetzlichen Maßnahmen zu treffen, die geeignet und nötig sind, beim Gefangenen das Vollzugsziel zu erreichen. Er hat auch die Aufgabe, die erforderlichen Mittel für den Personal- und Sachbedarf bereitzustellen (vgl. BVerfGE 36, 264 [275]).“30

Danach ist zwar einerseits kein unbegenzter Resourcenaufwand für den Vollzug zu verlangen (vgl. auch, zum gesetzgeberischen Entscheidungsspielraum hinsichtlich der Ausgestaltung des Strafvollzuges, B.I.2.). Andererseits gilt aber, dass Unterausstattung nicht anspruchsbegrenzend wirkt.

- „Verfassungsrechtliche Anforderungen an die Vollzugsgestaltung gelten nicht nur nach Maßgabe dessen, was an Verwaltungs- oder Justizeinrichtungentatsächlich oder üblicherweise vorhanden ist (vgl. BVerfGE 15, $288<296>$; 34, $369<380$ f.>; 40, $276<284>$; 116, 69 $<89$ f.>). Zwar können sich Grenzen für die Möglichkeit der Durchführung von Behandlungsmaßnahmen auch aus der räumlichen und personellen Ausstattung der Justizvollzugsanstalt ergeben (vgl. BVerfGE 42, $95<100 \mathrm{f}$. $>$ ). Der Strafgefangene kann nicht verlangen, dass unbegrenzt personelle und sonstige Mittel aufgewendet werden, um Beschränkungen seiner grundrechtlichen Freiheiten zu vermeiden (vgl.

30 BVerfGE 40, 276 (283 f.). 
BVerfGE 34, $369<380 \mathrm{f}>$; 34, $384<402>$; 35, $307<310>$; 42, 95 $<100$ f.>; BVerfGK 13, $163<166>$; 13, $487<492>$ ). Andererseits kann aber der Staat grundrechtliche und einfachgesetzlich begründete Ansprüche Gefangener nicht nach Belieben dadurch verkürzen, dass er die Vollzugsanstalten nicht so ausstattet, wie es zur Wahrung ihrer Rechte erforderlich wäre. Vielmehr setzen die Grundrechte auch Maßstäbe für die notwendige Beschaffenheit staatlicher Einrichtungen. Der Staat ist verpflichtet, Vollzugsanstalten in der zur Wahrung der Grundrechte erforderlichen Weise auszustatten (vgl. BVerfGE 40, 276 $<284>$; 45, $187<240>$; BVerfGK 13, $163<168$ f. $>$; $13,487<492$ f. $>$ m.w.N.)." ${ }^{11}$

Demgemäß genügt etwa zur Versagung von Lockerungen nicht die Berufung darauf, dass die personelle Ausstattung nichts anderes erlaube (vgl. BVerfGK 19, $157<163$ ff.>), das Recht eines Gefangenen zum Besitz von Gegenständen kann nicht nach Belieben mit dem Verweis auf einen damit verbundenen Überwachungsaufwand eingeschränkt werden (vgl. für die Untersuchungshaft BVerfG II/2, Beschluss vom 25.7.1994 - 2 BvR 806/94 -, juris; s. allerdings dazu, dass eine mit zumutbarem Kontrollaufwand nicht ausräumbare Gefahr für die Sicherheit oder Ordnung geeignet ist, Einschränkungen des Besitzes von Gegenständen im Haftraum zu rechtfertigen, B.XXI.1.). Gegebene räumliche Verhältnisse in einer Anstalt sind nicht geeignet, eine menschenwürdewidrige Haftraumunterbringung (BVerfGK 13, $472<479>$ ) oder beliebige Unterschiede in den Haftbedingungen von Männern und Frauen zu rechtfertigen (vgl. BVerfGK $14,381<386 \mathrm{f}$. $>$ ). Überbelegung oder Unterausstattung rechtfertigt auch nicht die Versagung angemessener Besuchskontakte mit Familienangehörigen (vgl. für die Untersuchungshaft BVerfGK 9, $365<370 \mathrm{f}$. $>$; BVerfG II/2, Beschluss vom 25.7.1994 - 2 BvR 806/94 -, juris; für die Strafhaft BVerfGK 13, $487<493$. >).

Bei Eingriffen, die den Intimbereich und das Schamgefühl des Inhaftierten berühren, ist besondere Rücksichtnahme geboten und der dazu erforderliche Aufwand hinzunehmen (BVerfG II/3, Beschluss vom 10.7.2013 - 2 BvR 2815/11 -, juris, Auszug unter B.XXV.).

Auf etwaige Engpässe müssen die Vollzugsbehörden mit besonderen Anstrengungen zur Vermeidung nicht hinnehmbarer Beeinträchtigungen reagieren, und die Gerichte sind dementsprechend prüfungspflichtig.

31 BVerfGK 19, 157 (163). 
- „Drohen aufgrund unzureichender Ausstattung von Haftanstalten Beeinträchtigungen, die normalerweise von Rechts wegen nicht hinnehmbar sind, so sind - unbeschadet der Pflicht der zuständigen Organe, für eine dauerhafte Verbesserung der Ausstattung zu sorgen - den zuständigen Anstalten und ihren Trägern besondere Anstrengungen zum Ausgleich des Mangels und zur zügigen Abhilfe abzuverlangen; das Niveau der "zumutbaren Anstrengungen" (vgl. BVerfGE 42, $95<102>$ ) bemisst sich insoweit nach der staatlichen Verantwortung für die Ausstattung des Vollzuges mit den für die rechtmäßige Erfüllung seiner Aufgaben erforderlichen Mitteln (vgl. BVerfGK 13, $487<493>$ ). Die hiernach entscheidungserheblichen Umstände haben die Gerichte aufzuklären. “32

„Unter anderem kann es bei Knappheit der räumlichen Ausstattung geboten sein, unter Einsatz des dazu notwendigen Personals eine übermäßige Beengtheit der Haftraumunterbringung durch entsprechend großzügige Aufschlusszeiten zu kompensieren (zu dieser Möglichkeit BVerfG, Beschluss der 2. Kammer des Zweiten Senats vom 13. November 2007 - 2 BvR 2354/04 -, EuGRZ 2008, S. 82), räumlich und personell bedingte Engpässe hinsichtlich der Ermöglichung von Besuchen durch den Einsatz von Überstunden auszugleichen (vgl. BVerfG, Beschluss der 2. Kammer des Zweiten Senats vom 25. Juli $1994-2$ BvR 806/94 -, NJW 1995, S. 1478 <1479>) und alle Möglichkeiten der Problementschärfung durch Verlegung von Gefangenen - soweit sich diese als das grundrechtsschonendere Mittel darstellt - auszuschöpfen (vgl. BVerfG, Beschluss der 2. Kammer des Zweiten Senats vom 13. November 2007 - 2 BvR 2201/05 -, www.bverfg.de; Beschluss der 2. Kammer des Zweiten Senats vom 16. März 1993, NStZ 1993, S. $404<406>$; zum Maßregelvollzug Beschluss der 2. Kammer des Zweiten Senats vom 13. November 2007 - 2 BvR 2354/04 -, EuGRZ 2008, S. 81; zur Untersuchungshaft Beschluss der 2. Kammer des Zweiten Senats vom 13. November 2007 - 2 BvR 939/07 -, EuGRZ 2008, S. 83).“33 
All dies betrifft auch die dem verfassungsrechtlichen Abstandsgebot für die Sicherungsverwahrung (s.u. C.I.) entsprechende bessere Ausstattung der Einrichtungen zum Vollzug der Sicherungsverwahrung.

- „Das Grundrecht aus Art. 2 Abs. 2 Satz 2 in Verbindung mit Art. 104 Abs. 1 Satz 1 GG verpflichtet den Staat, den Vollzug der Sicherungsverwahrung in deutlichem Abstand zum Strafvollzug auszugestalten (vgl. BVerfGE 109, $133<166$ f.>; 128, $326<375>$ ). ... Den grundrechtlichen Anspruch von Sicherungsverwahrten auf einen demgemäß ausgestalteten Vollzug kann der Staat - unabhängig davon, dass dem Gesetzgeber für eine entsprechende Neuregelung des Rechts der Sicherungsverwahrung eine Übergangsfrist eingeräumt ist (vgl. BVerfGE 128, $326 / /<332>$ ) - bereits gegenwärtig nicht nach freiem Belieben verkürzen. Die Grundrechte setzen Maßstäbe für die notwendige Beschaffenheit staatlicher Einrichtungen. Der Staat ist verpflichtet, Vollzugsanstalten in der zur Wahrung der Grundrechte erforderlichen Weise auszustatten (vgl. BVerfGE 40, $276<284>$; 45, $187<240>$; BVerfGK 13, $163<168$ f. $>$; 13, $487<492$ f. $>$ m.w.N.). Sind vorhandene Vollzugseinrichtungen und deren Ausstattung so beschaffen, dass Rechte der Gefangenen nicht gewahrt werden können, ohne dass dadurch Rechte anderer Gefangener oder sonstige Belange von vergleichbarem Gewicht beeinträchtigt werden, so folgt auch hieraus nicht, dass die insoweit auf der einen oder anderen Seite unvermeidlichen Beeinträchtigungen ohne weiteres und unabhängig von laufenden Bemühungen um kurzfristige Abhilfe als rechtmäßig hinzunehmen wären (vgl. BVerfGK 13, $487<493>$ m.w.N.). Die Frage, wie mit derartigen Notsituationen umzugehen ist, stellt sich im Übrigen erst, wenn feststeht, dass eine auch mit besonderem Einsatz nicht vermeidbare Notsituation tatsächlich vorliegt. Drohen aufgrund unzureichender Ausstattung von Haftanstalten Beeinträchtigungen, die normalerweise von Rechts wegen nicht hinnehmbar sind, so sind - unbeschadet der Pflicht der zuständigen Organe, für eine dauerhafte Verbesserung der Ausstattung zu sorgen - den zuständigen Anstalten und ihren Trägern besondere Anstrengungen zum Ausgleich des Mangels und zur zügigen Abhilfe abzuverlangen; das Niveau der "zumutbaren Anstrengungen" (vgl. BVerfGE 42, $95<102>$ ) bemisst sich insoweit nach der staatlichen Verantwortung für die Ausstattung des Vollzuges mit den für die recht- 
mäßige Erfüllung seiner Aufgaben erforderlichen Mitteln (vgl. BVerfGK 13, $487<493>$ ). “34

Bei der Bestimmung dessen, was einerseits Gefangenen an grundrechtsrelevanten Einschränkungen und andererseits dem Staat an Ressourcenaufwand zur Vermeidung oder Abmilderung solcher Einschränkungen zumutbar ist, muss hinsichtlich der Untersuchungsgefangenen berücksichtigt werden, dass diese noch nicht rechtskräftig verurteilt sind (BVerfGK $20,93<100>$ ).

Dass den Vollzugsanstalten keine Strategie der Mängelverwaltung ermöglicht werden darf, bei der Gefangene ohne wirksame Beanstandungsmöglichkeit rechtswidrigen Haftbedingungen ausgesetzt werden, ist im gerichtlichen Verfahren bei der Handhabung des Erfordernisses eines nach Erledigung fortbestehenden Rechtsschutzbedürfnisses zu berücksichtigen (BVerfGK 20, $249<256>$, Auszug unter E.II.10.d)). 